\title{
Comportamiento a flexión de vigas de madera reutilizada unida con bambú (Guadua angustifolia Kunth) para uso estructural
} Bending performance of beams made with reused
wood joined with bamboo (Guadua angustifolia
Kunth) for structural use

Andrés F. Guerra R. (')

Jorge E. Lozano P. (1)

(1) Universidad Nacional de Colombia afguerrari@unal.edu.co jelozanop@unal.edu.co

Recibido: 7 de Julio, 2019

Aceptado: 11 de Diciembre, 2019

Doi: http://dx.doi.org/10.24133/ciencia. v22i1.1288

"THIS IS AN OPEN ACCESS ARTICLE UNDER THE TERMS OF THE CREATIVE COMMONS ATTRIBUTION LICENSE, WHICH PERMITS USE, DISTRIBUTION AND REPRODUCTION IN ANY MEDIUM, PROVIDED THE ORIGINAL WORK IS PROPERLY CITED."

\begin{abstract}
RESUMEN
En esta investigación se estudió el comportamiento mecánico de vigas compuestas por piezas de madera reutilizada obtenida en procesos de deconstrucción unidas con láminas de Guadua angustifolia Kunth en la parte superior e inferior mediante el sistema de encolado prensado estructural, confinando la madera y asumiendo los mayores esfuerzos de tracción y compresión producidos por la flexión y el cortante. Para este propósito se realizaron ensayos según las normas ASTM D198 y NTC 5279 que permitieron hallar parámetros mecánicos como el esfuerzo máximo de las fibras de Guadua angustifolia Kunth en el límite proporcional, el módulo de rotura, el módulo de elasticidad y la ductilidad al desplazamiento. Como resultado se pudo determinar que el comportamiento mecánico del sistema no está en función de la densidad de las piezas de madera, el contenido de humedad y tamaño, sino de la resistencia mecánica de las fibras de Guadua angustifolia Kunth y la calidad de la línea de adhesivo. Así mismo se pudo determinar que las vigas tienen un comportamiento dúctil antes de llegar a la falla.
\end{abstract}

\section{Palabras claves:}

Madera reutilizada, bambú, Guadua angustifolia Kunth, vigas, construcción, sostenibilidad, deconstrucción.

\section{ABSTRACT}

This research studies the mechanical performance of beams composed of pieces of reused wood obtained in deconstruction processes, joined with Guadua angustifolia Kunth sheets in the upper and lower part with structural pressing glue system and confining the wood, assuming biggest stresses of traction and compression, produced by bending and shear efforts. For this purpose, tests were according to ASTM D198 and NTC5279 standards to find mechanical parameters such as the maximum stress of the Guadua angustifolia Kunth fibers in the proportional limit, the modulus of rupture, the 
modulus of elasticity and the ductility to displacement. The result was: the mechanical performance of the system is not a function of the density of the wood pieces, the moisture content and size, but of the mechanical strength of the Guadua angustifolia Kunth fibers and the quality of the line of adhesive. Likewise, it was possible to determine that the beams have a ductile performance before the fault.

\section{Keywords.}

Reused wood, bamboo, Guadua angustifolia Kunth, beams, construction, sustainability, deconstruction.

\section{INTRODUCCIÓN}

Para reducir los impactos ambientales que genera la industria de la construcción se proponen diferentes alternativas que controlen la extracción de materias primas no renovables y reduzcan la cantidad de desechos durante la construcción y la demolición de edificaciones. Una opción son los procesos de deconstrucción, en los cuales, los elementos que componen la edificación pueden ser desensamblados y reutilizados prolongando de la vida útil de los materiales recuperados, utilizándolos nuevamente sin que se requiera un proceso de transformación. Sin embargo, esta práctica se dificulta en construcciones que no fueron diseñadas para desensamblar sus elementos de manera fácil.

Los sistemas estructurales de madera permiten desensamblar sus componentes de manera sencilla, ya que sus uniones se basan en elementos metálicos como clavos, tornillos, pasadores y pletinas. Sin embargo, en una misma construcción se pueden recuperar piezas de madera de diferente especie, tamaño y calidad estructural.

En vista de la necesidad de buscar alternativas innovadoras para el aprovechamiento de la madera obtenida en procesos de construcción y demoliciones reduciendo la explotación incontrolada de este recurso y contribuir a la reforestación de los bosques, el Centro de Investigación de Bambú y Madera (CIBAM) y el Grupo de Investigación Madera y Guadua (GIMG) de la Universidad Nacional de Colombia, estudian el comportamiento mecánico de elementos estructurales construidos a partir de madera reciclada y reutilizada.

En esta investigación se determina el comportamiento mecánico a flexión de vigas construidas con piezas de madera reutilizada obtenida en procesos de deconstrucción, unidas y reforzadas con esterilla de bambú Guadua angustifolia Kunth mediante el sistema de laminado estructural prensado encolado.

El estudio experimental consistió en realizar ensayos de flexión a vigas de $3 \mathrm{~m}$ de longitud siguiendo los parámetros establecidos en la norma ASTM D198 - NTC5279. Encontrando el esfuerzo de la fibra en el límite proporcional, el módulo de rotura, el módulo de elasticidad y la ductilidad al desplazamiento de las vigas estudiadas.

Por otro lado, se examina el comportamiento de la línea de adhesivo en las probetas al corte longitudinal inducido por esfuerzos de flexión. Además, se analiza la resistencia y el módulo de elasticidad de las vigas en función del número de piezas, el contenido de humedad promedio, la densidad y la distribución de la madera reutilizada.

\section{VIGAS DE MADERA REUTILIZADA UNIDAS CON BAMBÚ MEDIANTE EL SISTEMA DE LAMINADO ESTRUCTURAL PRENSADO ENCOLADO.}

En esta investigación se recolectan piezas de madera producto de desmontes de estructuras y se lleva a unas dimensiones establecidas retirando los elementos metálicos, las imperfecciones y los residuos de otros materiales como adhesivos y morteros. Las piezas de madera son colocadas una junto a otra y se unen mediante una lámina superior e inferior de esterilla de Guadua angustifolia Kunth en el sistema de laminado encolado, 
confinando los elementos de madera reutilizada y asumiendo los esfuerzos máximos de compresión y tensión producto de la flexión (figura 1), este procedimiento se lleva a cabo para obtener elementos estructurales con características mecánicas de acuerdo a los parámetros del título $\mathrm{G}$ del código de construcción sismo resistente colombiano NSR10.

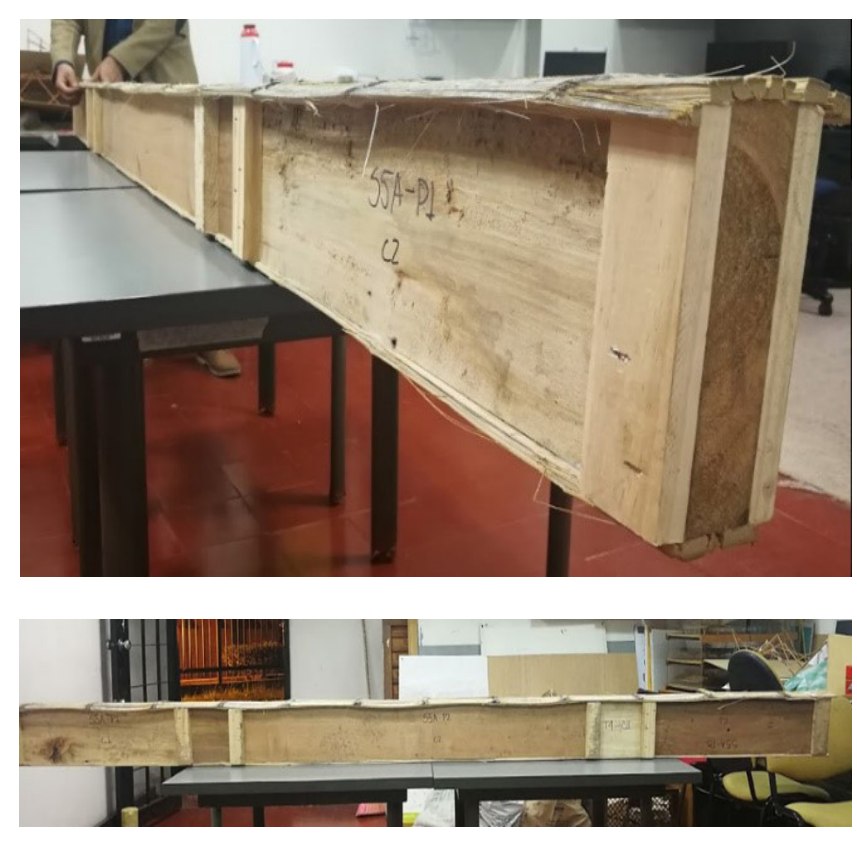

Figura 1. Vigas compuestas estudiadas

\subsection{DECONSTRUCCIÓN.}

Es una técnica en la cual se recuperan materiales y elementos de construcción durante el desmonte o demolición de una edificación, reduciendo costos de disposición y tratamiento de residuos y mitigando impactos ambientales generados en las etapas de extracción de materias primas, procesamiento y disposición final. (EPA, 2008)

En los procesos de deconstrucción, los materiales que componen a las edificaciones y que han llegado al final de su vida útil pueden ser reutilizados y reciclados utilizándolos como materia prima para la fabricación de nuevos elementos, haciendo que su ciclo de vida sea cerrado pasando de un análisis de ciclo de vida "creadle to grave" a uno "creadle to creadle" en el cual los materiales pueden ser recolectados, reutilizados o reciclados manteniendo una calidad aceptable. (McDonoufh \& Braungart, 2002)

El diseño para la deconstrucción (DfD) es un concepto en el cual, desde el diseño se proyectan las construcciones para que sus elementos se puedan desensamblar, reutilizar, manufacturar y reciclar en industrias. El principal objetivo de esta iniciativa es reducir los impactos en contaminación, aumentando la eficiencia económica y de los recursos naturales en remodelaciones y demoliciones de edificaciones recuperando sus componentes para extender su vida útil. (Guy, 2002)

\subsection{MADERA REUTILIZADA PARA USO ESTRUCTURAL.}

Los sistemas estructurales para la construcción en madera hacen que sus elementos sean fáciles de desmontar y reutilizar, sus conexiones se basan principalmente en elementos metálicos como clavos, tornillos pernos y pletinas. En deconstrucción los tamaños de la madera y la forma en que se unen y se cortan los elementos determinan su reutilización, las piezas más grandes tienen más opciones a la hora de evaluar sus posibles nuevos usos, mientras que en general los elementos pequeños contienen mayor cantidad de conectores como clavos y tornillos que se deben retirar manualmente consumiendo energía y tiempo. (Zabusova, 2014) 
Para llevar a cabo procesos de reutilización de madera es importante analizar las condiciones a las que estuvo expuesto el material en la edificación. (Flak et al., 1999)

La reutilización de elementos de madera a los que se les ha aplicado adhesivo, presentan una severa reducción en la resistencia cuando se vuelven a utilizar en productos encolados, en los elementos de madera reutilizada que tienen residuos de urea formaldehído se ve significativamente afectado el comportamiento del curado y la calidad de la adhesión del nuevo sistema, el mayor deterioro de la unión entre elementos se obtiene con mayor contenido y tamaño de residuos en la superficie de madera. (Zhong et al., 2017)

\subsection{MADERA LAMINADA ENCOLADA.}

La madera laminada se define como la unión de prismas de madera unidos mediante diferentes métodos, conformando un elemento monolítico que es continuo en escuadría y en largo. Dependiendo del tipo de unión los elementos laminares pueden ser pernados, calvados o encolados, por lo general las láminas se disponen a lo largo del eje longitudinal del elemento, sin embargo también se fabrican elementos laminados cruzados en donde las láminas adyacentes se ubican ortogonalmente, cabe aclarar que si las láminas son paralelas al plano neutro de flexión del elemento se considera un laminado horizontal, si son normales al plano neutro de flexión se trata de una laminación vertical. (Pérez, 1992)

En la fabricación de elementos estructurales de madera laminada se han utilizado dos clases de adhesivos, en un principio se usaron colas naturales producidas a base de materiales orgánicos y más adelante se implementaron los adhesivos sintéticos. Los adhesivos Orgánicos se crean a base de proteínas, estos adhesivos son muy susceptibles a la humedad generando restricciones en el uso estructural. Entre los adhesivos sintéticos se encuentran los Formaldehidos, usualmente son a base de fenol formaldehido (PF), resinol formaldehido (RF), fenol-resinol formaldehido (PRF), úrea formaldehído (UF) y melamina formaldehido. Estos polímeros se deben mezclar con catalizadores, cargas y disolventes para su aplicación, el fraguado de estos materiales se produce por la policondensación de la resina cuando reacciona con el catalizador, el tiempo de fraguado depende en gran parte de la temperatura ambiente, por lo general se realiza entre $15^{\circ} \mathrm{C}$ y $20^{\circ} \mathrm{C}$, para aumentar la velocidad del fraguado en procesos industriales se usan temperaturas entre los $70^{\circ} \mathrm{C}$ y $140^{\circ} \mathrm{C}$. (Quevedo, 2000)

\subsection{BAMBÚ Guadua angustifolia Kunth EN EL SISTEMA LAMINADO ESTRUCTURAL.}

La guadua se clasifica como un bambú leñoso del cual existen aproximadamente 1000 especies, 500 de estas especies presentes en América. El bambú Guadua angustifolia Kunth se encuentra principalmente en países como Colombia, Venezuela y Ecuador, aunque también existe en grandes cantidades en países centroamericanos como Panamá, que junto con Colombia se han caracterizado por el uso de esta especie en diferentes campos. (Olarte, 2012). Las fibras se encuentran orientadas axialmente dando a la guadua una alta resistencia a la tensión paralela a la fibra, siendo más resistente en la pared externa debido a su dureza y mayor cantidad de fibras, así mismo, la resistencia a la tensión se reduce en los nudos por la orientación de algunos haces vasculares. (Carvajal et al.,1981)

Para la Guadua angustifolia Kunth en el sistema laminado estructural la calidad y la durabilidad del elemento depende del adhesivo y su resistencia a las condiciones de humedad (Lozano, 2010). En cuanto a la determinación del mejor adhesivo para la fabricación de vigas laminadas de bambú Gonzalez et al., 2009 estudiaron diferentes tipos de pegantes entre ellos la melanina formaldehido, fenol resorcinol formaldehido y poliuretano, encontrando que para todos la falla se presentó en la Guadua angustifolia Kunth en la zona de interacción con el adhesivo, además la resistencia es menor en la cara interior de las tablillas, concluyendo que la falla no se produce en la línea de cola sino en el material blando de la guadua. Como conclusión de la investigación el adhesivo melanina formaldehido fue el mejor en cuanto a sostenibilidad y comportamiento mecánico. (González et al., 2009)

Vol. 22, No. 1:11-33 (2020) 


\section{ANÁLISIS EXPERIMENTAL}

\subsection{TRANSFORMACIÓN DE MATERIAS PRIMAS.}

\subsubsection{OBTENCIÓN Y PREPARACIÓN DE MADERA PARA SER REUTILIZADA.}

La madera utilizada en esta investigación fue recuperada de la estructura de cubierta de los edificios 238 y 239 ubicados dentro del campus de la Universidad Nacional de Colombia sede Bogotá. Durante este proceso los elementos de madera fueron desmontados tratando de mantener su integridad, sin embargo, algunos debieron ser cortados y otros sufrieron lesiones mecánicas. Cada pieza se estudió siguiendo los requisitos generales de calidad, según la clasificación visual por defectos establecida en título G - Estructuras de madera y estructuras de guadua del Reglamento Colombiano de Construcción Sismo Resistente NSR-10. Los elementos metálicos de fijación como clavos, pernos, platinas son retirados (figura 2), durante este proceso se pueden generar lesiones mecánicas como fisuras, grietas, abolladuras etc. que podrían hacer que el elemento sea descartado al no cumplir los requisitos de clasificación visual. Cada elemento de madera es cortado con una sierra eléctrica, perpendicularmente a su eje longitudinal, eliminando en lo posible defectos como nudos, perforaciones, pudrición, rajaduras y manchas. Mediante este proceso se obtuvieron piezas con escuadría mínima de $18 \mathrm{~cm}$ de altura, $4 \mathrm{~cm}$ de espesor. Para el largo de las piezas se definieron diferentes medidas, $260 \mathrm{~cm}, 130 \mathrm{~cm}, 86$ $\mathrm{cm}$ y $65 \mathrm{~cm}$.

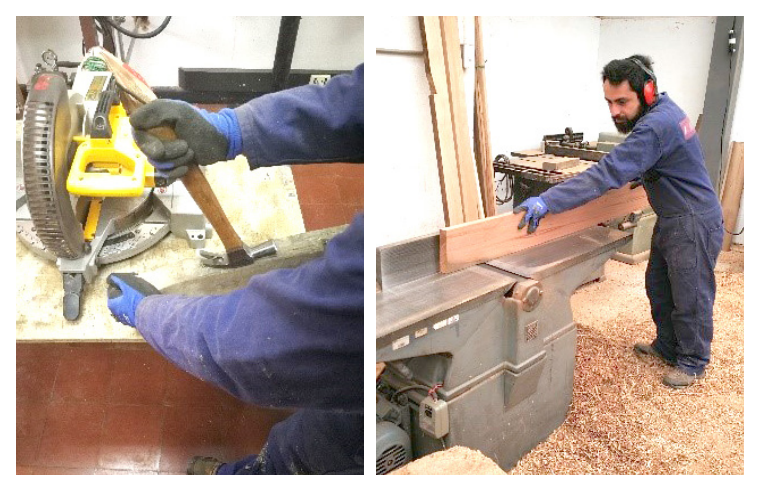

Figura 2. Retiro de elementos metálicos.

Las piezas se dejan planas por una de las caras de $18 \mathrm{~cm}$ y se deja ortogonal una de las caras de $4 \mathrm{~cm}$. Este proceso se lleva a cabo utilizando una planeadora para madera. Luego se procede a cepillar cada elemento con un cepillo eléctrico para madera (figura 3), con el cual se logra garantizar un espesor uniforme para todas las piezas.
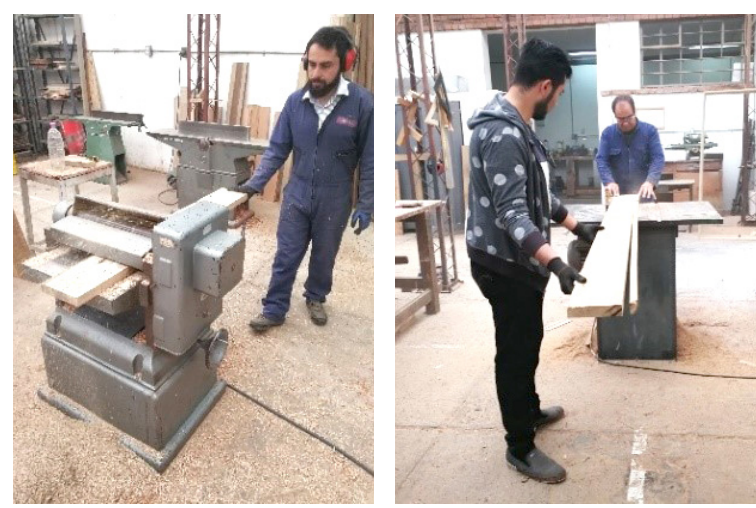

Figura 3. Proceso de cepillado y corte.

Para identificar las diferentes especies de madera se realiza una caracterización teniendo en cuenta las características macroscópicas de la madera y las propiedades organolépticas, como el olor y la apariencia, teniendo 
en cuenta detalles sobre su estructura realizando observaciones mediante una lupa o un estéreo microscopio, determinando un conjunto de características xilemáticas en los elementos de la estructura celular y propiedades físico químicas.

\subsubsection{OBTENCIÓN Y PREPARACIÓN DEL BAMBÚ Guadua angustifolia Kunth}

La Guadua angustifolia Kunth que se utilizó en la construcción de las vigas se obtuvo en la región de El Socorro, Santander - Colombia, municipio ubicado sobre la cordillera oriental del sistema montañoso de los Andes, esta región tiene una temperatura media de $23^{\circ} \mathrm{C}$, y se encuentra a una altitud media de $1.300 \mathrm{~m}$. sobre el nivel del mar, el relieve varía entre fuertemente escarpado con pendientes entre el $25 \%$ y $50 \%$, al muy escarpado con pendientes mayores al $75 \%$. Fueron seleccionadas Guaduas que no cumplen algunos requisitos mínimos de clasificación visual por defectos descrito en el titulo - G, G.12.3.2 del NSR10, pero que a su vez no afecta el diseño de los elementos estructurales estudiados en esta investigación ya que no se utilizara en su forma cilíndrica, esta selección contribuye a optimizar el aprovechamiento de los guaduales y además reduce costos de fabricación.

Para elaborar las láminas de esterilla que se colocan en la parte superior e inferior de las vigas, se realizan incisiones en el nudo de forma paralela al eje longitudinal de la Guadua, la separación entre cada corte se realiza cada $3 \mathrm{~cm}$ aproximadamente, este procedimiento se lleva a cabo generando fisuras en los entrenudos. Posteriormente se abre la Guadua longitudinalmente y se retira la parte interna de los nudos. Además, se debe retirar el tejido parenquimático que cubre las fibras de la parte interior del culmo para garantizar la adhesión entre la madera y las fibras de guadua con el adhesivo, este procedimiento se realiza con una lijadora de banda, desbastando la parte interior de la esterilla hasta que las fibras queden expuestas, así mismo es necesario retirar las ramas en la parte externa ya que en el prensado produce rotaciones de la viga (figura 4).
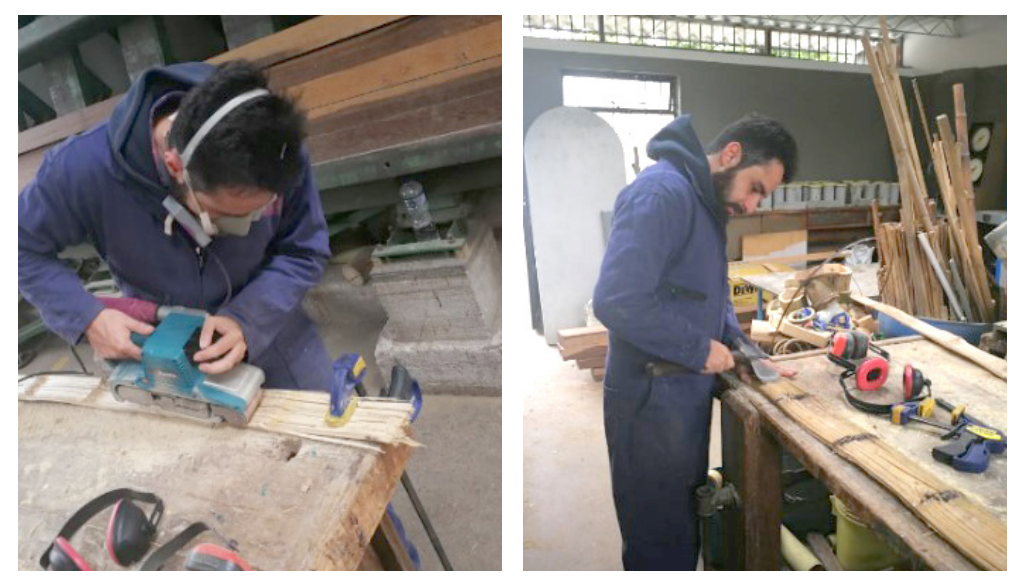

Figura 4. Retiro de tejiro parenquimatico y nudos.

\subsection{CONSTRUCCIÓN DE VIGAS.}

Se lleva a cabo en dos procesos, ensamble del alma de las vigas y el encolado y prensado del alma con las láminas de Guadua angustifolia Kunth.

\subsubsection{ENSAMBLE DE PIEZAS DE MADERA PARA EL ALMA DE LAS VIGAS.}

Las piezas de madera tienen una altura de $16 \mathrm{~cm}$, esta altura corresponde a la máxima dimensión que se pudo recuperar teniendo en cuenta que todas las piezas deben tener la misma altura para garantizar la presión de prensado constante a lo largo del elemento. En el sentido longitudinal, se usan diferentes dimensiones debido a que en algunos elementos es necesario realizar cortes en el proceso de deconstrucción y para eliminar defectos. Para mantener uniforme la distribución de las piezas en esta investigación se logra recuperar longitudes de $260 \mathrm{~cm}, 130$ $\mathrm{cm}, 86 \mathrm{~cm}$ y $65 \mathrm{~cm}$. 
Se construyen 12 vigas con 6 configuraciones diferentes, en función de la longitud de las piezas. Para mantener los elementos de madera unidos durante el proceso de encolado y para garantizar la continuidad de las fibras de guadua que quedan fuera del alma, se utilizan uniones de madera de $16 \mathrm{~cm}$ de altura, $5 \mathrm{~cm}$ de ancho y $1.9 \mathrm{~cm}$ de espesor, unidos a la madera reutilizada mediante grapas. No se utiliza adhesivo para unir las piezas de madera.

\subsubsection{ENCOLADO Y PRENSADO DEL ALMA CON LAS LÁMINAS DE Guadua angustifolia Kunth.}

El adhesivo utilizado para adherir la esterilla de Guadua angustifolia Kunth es úrea melamina formaldehído (MUF1442), este polímero termoestable debe mezclarse con un catalizador (Hardener 2542) para originar la policondensación de la resina y producir el fraguado, el tiempo de fraguado está determinado por la temperatura en el momento de la aplicación de la mezcla. Se debe garantiza una presión uniforme en los elementos a adherir durante un tiempo determinado por la dosificación de la mezcla (figura 5), luego curan los elementos pegados durante un tiempo definido por la temperatura ambiente.

Teniendo en cuenta que la madera pierde rigidez y resistencia al aumentar el contenido de humedad, se mide el contenido de humedad de cada madera antes de realizar el ensayo de flexión.

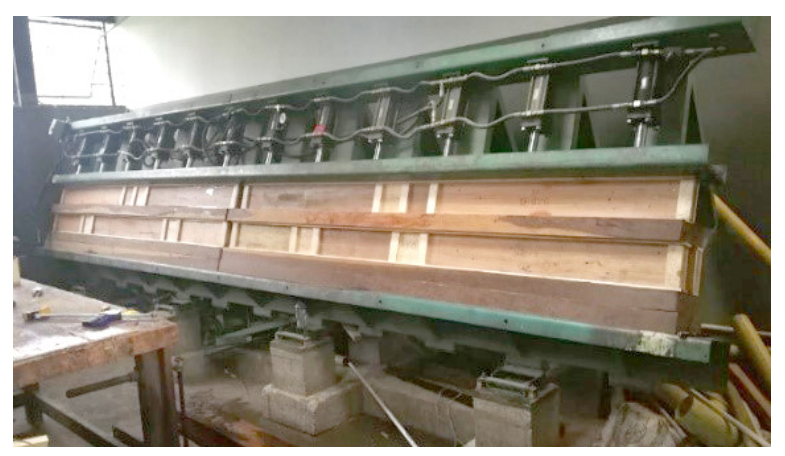

Figura 5. Proceso de prensado.

\subsection{ENSAYOS DE FLEXIÓN PARA MADERA EN TAMAÑOS ESTRUCTURALES ASTM D198 - NTC5279.}

En este método la viga se somete a un momento flector mientras se sostiene cerca de sus extremos en apoyos llamados reacciones y se le aplican dos cargas transversales impuestas simétricamente entre las reacciones (figura 6). La viga se deforma a una velocidad constante. El ensayo se realiza hasta la ruptura, se toman mediciones de la deflexión de la viga en el centro de la luz con un comparador de caratula hasta alcanzar la deformación máxima permitida en las deflexiones admisibles contempladas en el titulo G del Reglamento Colombiano de Construcción Sismo Resistente NSR10.

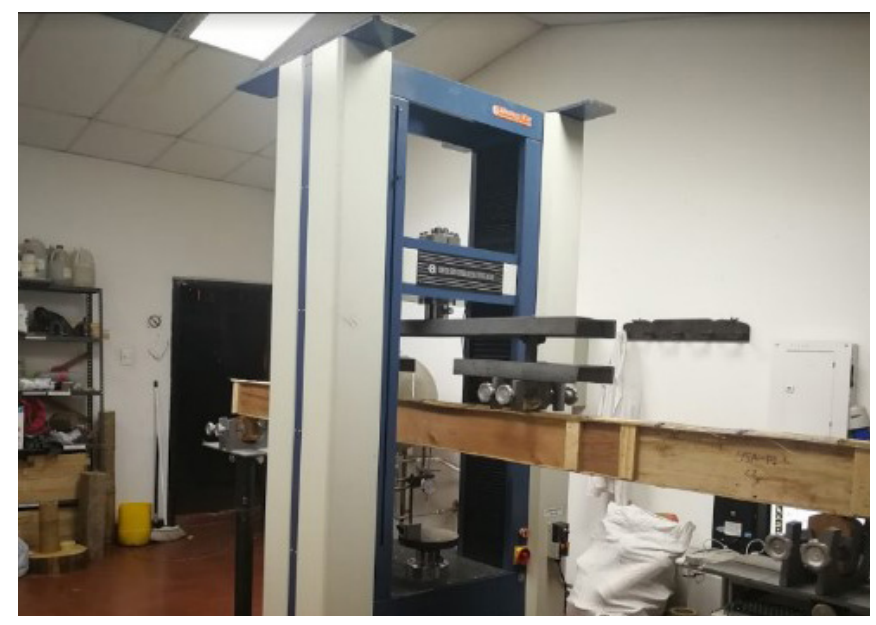

Figura 6. Ensayo de flexion. 
Para cada viga se registraron los valores de fuerza ( $\mathrm{kgf}$ ) y desplazamiento ( $\mathrm{mm}$ ) (figura 7)

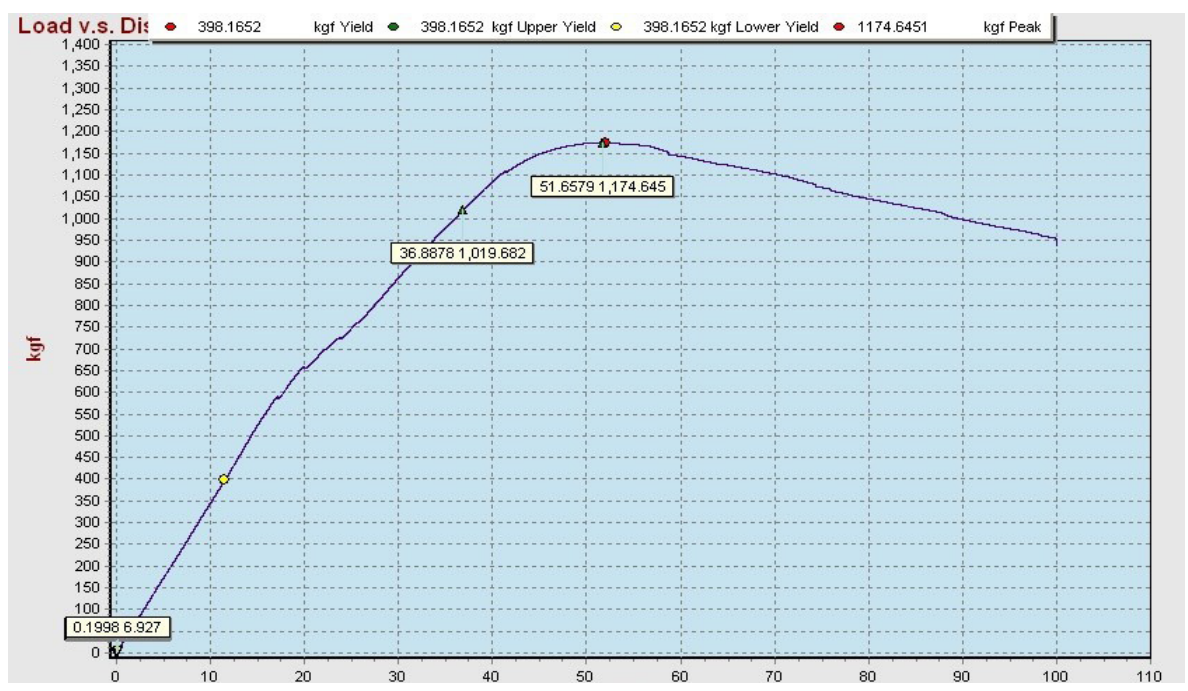

Figura 7. Curvas carga (kgf) desplazamiento $(\mathrm{mm})$.

Con los datos obtenidos en el ensayo de flexión siguiendo los parámetros descritos en las normas ASTM D198 NTC5279 se realiza el cálculo del esfuerzo cortante y momento flector máximo para cada probeta.

\subsubsection{CORTANTEMOMENTO FLECTOR YDEFLEXIÓN PARA LA CARGAMÁXIMA YLÍMITEPROPORCIONAL.}

Tabla 1.

Esfuerzo cortante, momento y deflexion para la carga maxima y para carga en el limite proporcional

\begin{tabular}{|c|c|c|c|c|}
\hline Probeta & $\begin{array}{c}\text { Carga } \\
\text { máxima } \\
P(\mathbf{N})\end{array}$ & $\begin{array}{c}\text { Cortante } \\
\text { máximo } \\
\boldsymbol{V}(\mathbf{N})\end{array}$ & $\begin{array}{c}\text { Momento } \\
\text { máximo } \\
M\left(\mathbf{N}^{*} \mathbf{m m}\right)\end{array}$ & $\begin{array}{c}\text { Deflexión } \\
\Delta(\mathbf{m m})\end{array}$ \\
\hline G1A & 11519.33 & 5759.67 & 5414086.22 & 48.56 \\
\hline G1B & 14482.00 & 7241.00 & 6806539.91 & 41.53 \\
\hline G2A & 5067.00 & 2533.50 & 2381489.05 & 30.53 \\
\hline G2B & 3985.47 & 1992.74 & 1873171.65 & 18.13 \\
\hline G3A & 2353.30 & 1176.65 & 1106051.85 & 7.264 \\
\hline G3B & 3530.67 & 1765.33 & 1659414.24 & 24.34 \\
\hline G4A & 4861.00 & 2430.50 & 2284669.76 & 26.17 \\
\hline G4B & 6367.33 & 3183.67 & 2992645.27 & 47.23 \\
\hline G5A & 5560.00 & 2780.00 & 2613199.01 & 25.11 \\
\hline G5B & 7966.33 & 3983.17 & 3744177.01 & 35.63 \\
\hline G6A & 2850.26 & 1425.13 & 1339623.89 & 10.12 \\
\hline G6B & 6259.33 & 3129.66 & 2941884.97 & 35.38 \\
\hline
\end{tabular}

\begin{tabular}{|c|c|c|c|c|}
\hline Probeta & $\begin{array}{c}\text { Carga en el límite } \\
\text { proporcional } \\
\boldsymbol{P I}(\mathbf{N})\end{array}$ & $\begin{array}{c}\text { Cortante } \\
\text { VIp(N) }\end{array}$ & $\begin{array}{c}\text { Momento } \\
\left.\text { MIp( } \mathbf{N}^{*} \mathbf{m m}\right)\end{array}$ & $\begin{array}{c}\text { Deflexión } \\
\Delta(\mathbf{m m})\end{array}$ \\
\hline G1A & 10787.32 & 5393.66 & 5070038.05 & 37.72 \\
\hline G1B & 13729.31 & 6864.66 & 6452775.70 & 39.19 \\
\hline G2A & 4314.93 & 2157.46 & 2028015.22 & 14.70 \\
\hline G2B & 1916.22 & 958.11 & 900623.12 & 6.74 \\
\hline G3A & 2353.30 & 1176.65 & 1106051.85 & 7.26 \\
\hline G3B & 2458.33 & 1229.17 & 1155415.58 & 15.02 \\
\hline G4A & 4860.96 & 2430.48 & 2284651.33 & 26.24 \\
\hline G4B & 4917.64 & 2458.82 & 2311292.07 & 25.00 \\
\hline G5A & 4400.93 & 2200.47 & 2068437.25 & 17.42 \\
\hline G5B & 3115.96 & 1557.98 & 1464503.54 & 10.30 \\
\hline G6A & 2850.26 & 1425.13 & 1339623.89 & 10.12 \\
\hline G6B & 3143.82 & 1571.91 & 1477593.45 & 10.48 \\
\hline
\end{tabular}

\subsubsection{CÁlCULO DE LA CONSTANTE DE ELASTICIDAD EN EL ALMA CE POR EL MÉTOdO DE LOS ELEMENTOS FINITOS.}

A partir de los valores de fuerza y desplazamiento obtenidos en el ensayo de flexión dentro del rango elástico de la viga, se determina la constante de elasticidad en el alma compuesta por distintas especies de madera reutilizada con diferentes dimensiones, propiedades mecánicas y contenidos de humedad, estos elementos no están unidos con adhesivo provocando desplazamientos relativos perpendiculares al eje neutro de la sección al vencerse la fuerza de fricción entre ellos y al deformarse la esterilla de guadua que compone los patines de la viga. Es necesario conocer la tasa de deformación que determina el comportamiento del alma dentro del rango elástico, para calcular un factor $\mathrm{n}$ que relaciona el módulo de elasticidad de la guadua y la tasa de deformación en el alma permitiendo calcular la ubicación del eje neutro y el momento de inercia de una sección transformada. 


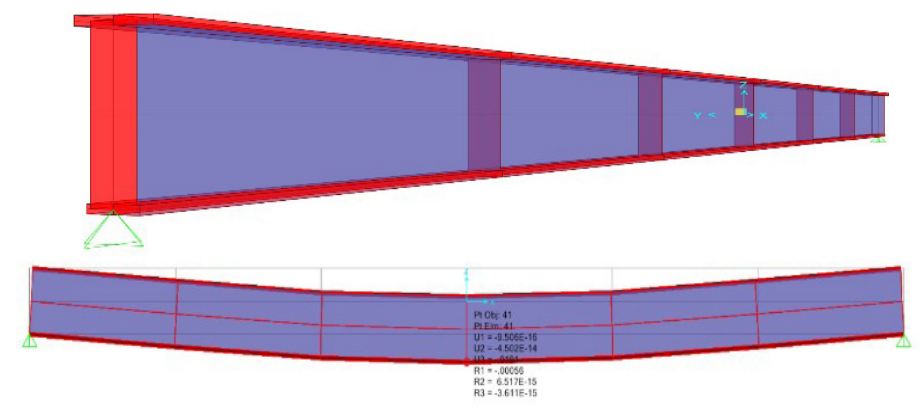

Figura 8. Modelo de elementos finitos.

Para conocer la constante de elasticidad del alma se realiza un modelo de elementos finitos de cada viga en las condiciones del ensayo de flexión para la carga registrada en los $8 \mathrm{~mm}$ de deformación (figura 8), el módulo de elasticidad del alma será ajustado mediante iteraciones hasta alcanzar la deformación de los 8 mm. (Tabla 2).

Tabla 2. Constante de elasticidad del alma de las vigas.

\begin{tabular}{|c|c|c|c|c|c|}
\hline PROBETA & Carga (kgf) & $\begin{array}{c}\text { Carga por bloque } \\
\text { de carga (kgf) }\end{array}$ & $\begin{array}{c}\text { Deflexión } \\
\Delta(\mathbf{m m})\end{array}$ & $\begin{array}{c}\text { Constante de elasticidad } \\
\text { del alma CE (kgf/m²) }\end{array}$ & $\begin{array}{c}\text { Constante de elasticidad } \\
\text { del alma CE (MPa) }\end{array}$ \\
\hline G1A & 386.4 & 193.2 & 8 & 125000000.00 & 1225.83 \\
\hline G1B & 382.9 & 191.45 & 8 & 125000000.00 & 1225.83 \\
\hline G2A & 272.8 & 136.4 & 8 & 33000000.00 & 323.62 \\
\hline G2B & 197.8 & 98.9 & 7 & 16000000.00 & 156.91 \\
\hline G3A & 234.37 & 117.185 & 7.5 & 23000000.00 & 225.55 \\
\hline G3B & 177.93 & 88.965 & 8 & 8300000.00 & 81.39 \\
\hline G4A & 279.2 & 139.6 & 8 & 35000000.00 & 343.23 \\
\hline G4B & 273.6 & 136.8 & 8 & 32000000.00 & 313.81 \\
\hline G5A & 287.53 & 143.765 & 8 & 41000000.00 & 402.07 \\
\hline G5B & 271.87 & 135.935 & 8 & 32000000.00 & 313.81 \\
\hline G6A & 240.13 & 120.065 & 8 & 20000000.00 & 196.13 \\
\hline G6B & 280.08 & 140.04 & 8 & 36000000.00 & 353.04 \\
\hline
\end{tabular}

\subsubsection{CÁLCULO DE LA UBICACIÓN DEL EJE NEUTRO Y MOMENTO DE INERCIA I DE LA SECCIÓN.}

Para calcular la ubicación del eje neutro y el momento de inercia de cada elemento es necesario analizar cada tipo de viga como un material heterogéneo de sección compuesta. Por lo tanto, es necesario realizar cálculos sobre una sección transformada asumiendo que toda la viga tiene el MOE de la Guadua angustifolia Khunth. (Tabla 3)

Tabla 3. Momento de inercia seccion transformada

\begin{tabular}{|c|c|c|c|c|c|c|c|c|c|c|c|c|c|c|c|c|c|}
\hline & \multicolumn{6}{|c|}{ PATIN SUPERIOR } & \multicolumn{5}{|c|}{ ALMA SECCION TRANSFORMADA } & \multicolumn{5}{|c|}{ PATIN SUPERIOR } & \multirow[b]{2}{*}{$\begin{array}{l}\text { Iv } \\
(\mathrm{mm} 4)\end{array}$} \\
\hline & $\begin{array}{c}\text { Ye } \\
(\mathrm{mm})\end{array}$ & $\begin{array}{c}\mathbf{b} \\
(\mathrm{mm})\end{array}$ & $\underset{(\mathrm{mm})}{\mathrm{h}}$ & $\underset{\left(\mathrm{mm}^{2}\right)}{\mathbf{A}}$ & $\underset{\left(\mathrm{mm}^{4}\right)}{\mathrm{I}}$ & $\begin{array}{c}\mathrm{Y} \\
(\mathrm{mm})\end{array}$ & $\begin{array}{c}\text { bt } \\
(\mathrm{mm})\end{array}$ & $\begin{array}{c}\mathrm{h} \\
(\mathrm{mm})\end{array}$ & $\underset{\left(\mathrm{mm}^{2}\right)}{\mathbf{A}}$ & $\underset{\left(\mathrm{mm}^{4}\right)}{\mathrm{I}}$ & $\begin{array}{c}Y \\
(\mathrm{~mm})\end{array}$ & $\underset{(\mathrm{mm})}{\mathbf{b}}$ & $\underset{(\mathrm{mm})}{\mathrm{h}}$ & $\underset{\left(\mathrm{mm}^{2}\right)}{\mathbf{A}}$ & $\underset{\left(\mathrm{mm}^{4}\right)}{\mathrm{I}}$ & $\begin{array}{c}Y \\
(\mathrm{~mm})\end{array}$ & \\
\hline G1A & 99.87 & 60 & 10 & 600 & 5000 & 175 & 4.52 & 160 & 723.20 & 200000 & 90 & 40 & 10 & 400 & 3333.3 & 5 & 8607091.56 \\
\hline G1B & 99.87 & 60 & 10 & 600 & 5000 & 175 & 4.52 & 160 & 723.20 & 200000 & 90 & 40 & 10 & 400 & 3333.3 & 5 & 8607091.56 \\
\hline G2A & 104.28 & 60 & 10 & 600 & 5000 & 175 & 1.19 & 160 & 190.40 & 406964 & 90 & 40 & 10 & 400 & 3333.3 & 5 & 7397595.62 \\
\hline G2B & 105.56 & 60 & 10 & 600 & 5000 & 175 & 0.58 & 160 & 92.80 & 197316 & 90 & 40 & 10 & 400 & 3333.3 & 5 & 7166116.11 \\
\hline G3A & 105 & 60 & 10 & 600 & 5000 & 175 & 0.83 & 160 & 132.80 & 283641 & 90 & 40 & 10 & 400 & 3333.3 & 5 & 7261889.88 \\
\hline G3B & 106.22 & 60 & 10 & 600 & 5000 & 175 & 0.3 & 160 & 48.00 & 102358 & 90 & 40 & 10 & 400 & 3333.3 & 5 & 7059922.24 \\
\hline G4A & 104.14 & 60 & 10 & 600 & 5000 & 175 & 1.26 & 160 & 201.60 & 431628 & 90 & 40 & 10 & 400 & 3333.3 & 5 & 7424593.88 \\
\hline G4B & 104.35 & 60 & 10 & 600 & 5000 & 175 & 1.16 & 160 & 185.60 & 394631 & 90 & 40 & 10 & 400 & 3333.3 & 5 & 7384079.42 \\
\hline G5A & 103.74 & 60 & 10 & 600 & 5000 & 175 & 1.48 & 160 & 236.80 & 505621 & 90 & 40 & 10 & 400 & 3333.3 & 5 & 7505326.89 \\
\hline G5B & 104.35 & 60 & 10 & 600 & 5000 & 175 & 1.16 & 160 & 185.60 & 394631 & 90 & 40 & 10 & 400 & 3333.3 & 5 & 734079.42 \\
\hline G6A & 105.24 & 60 & 10 & 600 & 5000 & 175 & 0.72 & 160 & 115.20 & 246645 & 90 & 40 & 10 & 400 & 3333.3 & 5 & 7220927.9 \\
\hline G6B & 104.07 & 60 & 10 & 600 & 5000 & 175 & 1.3 & 160 & 208.00 & 443960 & 90 & 40 & 10 & 400 & 3333.3 & 5 & 7438076.25 \\
\hline
\end{tabular}




\subsubsection{CÁLCULO DEL ESFUERZO MÁXIMO EN LA FIBRA EN EL LÍMITE PROPORCIONAL SF.}

El esfuerzo máximo de tensión de las fibras en el límite proporcional se calcula mediante la siguiente ecuación:

$$
S_{f t}=\frac{M_{l p} * C_{t}}{I_{v}}
$$

\footnotetext{
$\mathrm{Sft}=\quad$ Esfuerzo de tensión de la fibra en el límite proporcional.

Mlp $=$ Momento para la carga registrada en el límite proporcional.

$\mathrm{Ct}=\quad$ Distancia del eje neutro a la fibra más alejada sometida a tensión.

IV $=\quad$ Momento de inercia de la viga.
}

Tabla 4.

Esfuerzo maximo de tension de la fibra en el limite proporcional

\begin{tabular}{|c|c|c|c|c|}
\hline PROBETA & $\begin{array}{c}\text { Momento } \\
\text { Mlp(N*mm) }\end{array}$ & $\mathbf{C t}(\mathbf{m m})$ & $\mathbf{I v}\left(\mathbf{m m}^{4}\right)$ & Sft (MPa) \\
\hline G1A & 5070038.05 & 99.87 & 8607091.56 & 58.83 \\
\hline G1B & 6452775.70 & 99.87 & 8607091.56 & 74.87 \\
\hline G2A & 2028015.22 & 104.28 & 7397595.62 & 28.59 \\
\hline G2B & 900623.12 & 105.56 & 7166116.11 & 13.27 \\
\hline G3A & 1106051.85 & 105 & 7261889.88 & 15.99 \\
\hline G3B & 1155415.58 & 106.22 & 7059922.24 & 17.38 \\
\hline G4A & 2284651.33 & 104.14 & 7424593.88 & 32.05 \\
\hline G4B & 2311292.07 & 104.35 & 7384079.42 & 32.66 \\
\hline G5A & 2068437.25 & 103.74 & 7505326.89 & 28.59 \\
\hline G5B & 1464503.54 & 104.35 & 7384079.42 & 20.7 \\
\hline G6A & 1339623.89 & 105.24 & 7220927.9 & 19.52 \\
\hline G6B & 1477593.45 & 104.07 & 7438076.25 & 20.67 \\
\hline
\end{tabular}

Por otro lado el esfuerzo máximo de compresión de las fibras en el límite proporcional se calcula así:

$$
S_{f c}=\frac{M_{l p} * C_{c}}{I_{v}}
$$

$S_{f c}=$ Esfuerzo de compresión de la fibra en el límite proporcional.

$M_{l p}=$ Momento para la carga registrada en el límite proporcional.

$C_{c}=$ Distancia del eje neutro a la fibra más alejada sometida a compresión.

$I_{v}=$ Momento de inercia de la viga. 
Tabla 5.

Esfuerzo maximo de compresión de la fibra en el limite proporcional

\begin{tabular}{|c|c|c|c|c|}
\hline Probeta & Momento $\mathbf{M} I_{p}\left(\mathbf{N}^{*} \mathrm{~mm}\right)$ & $\mathbf{C}_{c}(\mathbf{m m})$ & $I_{v}\left(\mathbf{m m}^{4}\right)$ & $S_{f c}(\mathbf{M P a})$ \\
\hline G1A & 5070038.05 & 80.13 & 8607091.56 & 47.2 \\
\hline G1B & 6452775.7 & 80.13 & 8607091.56 & 60.07 \\
\hline G2A & 2028015.22 & 75.72 & 7397595.62 & 20.76 \\
\hline G2B & 900623.1227 & 74.44 & 7166116.11 & 9.36 \\
\hline G3A & 1106051.846 & 75.00 & 7261889.88 & 11.42 \\
\hline G3B & 1155415.58 & 73.78 & 7059922.24 & 12.07 \\
\hline G4A & 2284651.328 & 75.86 & 7424593.88 & 23.34 \\
\hline G4B & 2311292.073 & 75.65 & 7384079.42 & 23.68 \\
\hline G5A & 2068437.251 & 76.26 & 7505326.89 & 21.02 \\
\hline G5B & 1464503.536 & 75.65 & 7384079.42 & 15.00 \\
\hline G6A & 1339623.89 & 74.76 & 7220927.90 & 13.87 \\
\hline G6B & 1477593.453 & 75.93 & 7438076.25 & 15.08 \\
\hline
\end{tabular}

\subsubsection{CÁLCULO DEL MÓDULO DE RUPTURA SR.}

El módulo de ruptura para las fibras en tensión se calcula con la siguiente ecuación:

$$
S_{R t}=\frac{M * C_{t}}{I_{v}}
$$

$S_{R t}=$ Esfuerzo de tensión de la fibra en el límite proporcional.

$M=$ Momento para la carga máxima.

$C_{t}=$ Distancia del eje neutro a la fibra más alejada sometida a tensión.

$I_{v}=$ Momento de inercia de la viga.

Tabla 6.

Módulo de rotura fibras a compresión.

\begin{tabular}{|c|c|c|c|c|}
\hline Probeta & Momento $\mathbf{M}\left(\mathbf{N}^{*} \mathbf{m m}\right)$ & $\mathbf{C}_{\mathbf{t}}(\mathbf{m m})$ & $I_{v}\left(\mathbf{m m}^{4}\right)$ & $\mathbf{S}_{R t}(\mathbf{M P a})$ \\
\hline G1A & 5414086.22 & 99.87 & 8607091.56 & 62.82 \\
\hline G1B & 6806539.91 & 99.87 & 8607091.56 & 78.98 \\
\hline G2A & 2381489.05 & 104.28 & 7397595.62 & 33.57 \\
\hline G2B & 1873171.65 & 105.56 & 7166116.11 & 27.59 \\
\hline G3A & 1106051.85 & 105.00 & 7261889.88 & 15.99 \\
\hline G3B & 1659414.24 & 106.22 & 7059922.24 & 24.97 \\
\hline G4A & 2284669.76 & 104.14 & 7424593.88 & 32.05 \\
\hline G4B & 2992645.27 & 104.35 & 7384079.42 & 42.29 \\
\hline G5A & 2613199.01 & 103.74 & 7505326.89 & 36.12 \\
\hline G5B & 3744177.01 & 104.35 & 7384079.42 & 52.91 \\
\hline G6A & 1339623.89 & 105.24 & 7220927.90 & 19.52 \\
\hline G6B & 2941884.97 & 104.07 & 7438076.25 & 41.16 \\
\hline
\end{tabular}

Por otro lado el módulo de ruptura para las fibras en compresión calcula así:

$$
S_{R c}=\frac{M * C_{c}}{I_{v}}
$$


$S_{R c}=$ Esfuerzo de compresión de la fibra en el límite proporcional.

$M=$ Momento para la carga registrada en el límite proporcional.

$C_{c}=$ Distancia del eje neutro a la fibra más alejada sometida a compresión.

$I_{v}=$ Momento de inercia de la viga.

Tabla 7.

Módulo de rotura fibras a tension.

\begin{tabular}{|c|c|c|c|c|}
\hline Probeta & Momento $\mathbf{M}\left(\mathbf{N}^{*} \mathbf{m m}\right)$ & $\mathbf{C}_{\mathrm{c}}(\mathbf{m m})$ & $\mathrm{I}_{\mathrm{v}}\left(\mathbf{m m}^{4}\right)$ & $\mathbf{S}_{\mathrm{Rc}}(\mathbf{M P a})$ \\
\hline G1A & 5414086.22 & 80.13 & 8607091.56 & 50.40 \\
\hline G1B & 6806539.91 & 80.13 & 8607091.56 & 63.37 \\
\hline G2A & 2381489.05 & 75.72 & 7397595.62 & 24.38 \\
\hline G2B & 1873171.65 & 74.44 & 7166116.11 & 19.46 \\
\hline G3A & 1106051.85 & 75.00 & 7261889.88 & 11.42 \\
\hline G3B & 1659414.24 & 73.78 & 7059922.24 & 17.34 \\
\hline G4A & 2284669.76 & 75.86 & 7424593.88 & 23.34 \\
\hline G4B & 2992645.27 & 75.65 & 7384079.42 & 30.66 \\
\hline G5A & 2613199.01 & 76.26 & 7505326.89 & 26.55 \\
\hline G5B & 3744177.01 & 75.65 & 7384079.42 & 38.36 \\
\hline G6A & 1339623.89 & 74.76 & 7220927.90 & 13.87 \\
\hline G6B & 2941884.97 & 75.93 & 7438076.25 & 30.03 \\
\hline
\end{tabular}

\subsubsection{CÁLCULO DEL MÓDULO DE ELASTICIDAD E}

Para hallar el módulo de elasticidad de cada viga se despeja E, de la ecuación de deflexión que para una viga simplemente apoyada con dos cargas puntuales ubicadas simétricamente la deflexión en el centro de la luz es:

$$
E=\frac{P * a}{24 \Delta I} *\left(3 L^{2}-4 a^{2}\right)
$$

$\mathrm{E}=$ Módulo de elasticidad MOE.

$\Delta=$ Deflexión.

$\mathrm{P}=$ Fuerza.

$a=$ Distancia entre la reacción y el punto de carga más cercano.

$\mathrm{I}=$ Momento de inercia de la viga.

$L=\quad$ Luz, distancia entre las reacciones.

Tabla 8.

Módulo de elasticidad.

\begin{tabular}{|c|c|c|c|c|c|c|}
\hline Probeta & Carga límite proporcional $(\mathbf{N})$ & $\Delta$ límite proporcional $(\mathbf{m m})$ & $\mathbf{a}(\mathbf{m m})$ & $\mathbf{L}(\mathbf{m m})$ & $I_{v}\left(\mathbf{m m}^{4}\right)$ & $E(\mathbf{M P a})$ \\
\hline G1A & 10787.32 & 37.72 & 940.00 & 2820.00 & 8607091.56 & 26447.55 \\
\hline G1B & 13729.31 & 39.19 & 940.00 & 2820.00 & 8607091.56 & 32397.92 \\
\hline G2A & 4314.93 & 14.70 & 940.00 & 2820.00 & 7397595.62 & 31583.89 \\
\hline G2B & 1916.22 & 6.74 & 940.00 & 2820.00 & 7166116.11 & 31579.24 \\
\hline G3A & 2353.30 & 7.26 & 940.00 & 2820.00 & 7261889.88 & 35510.13 \\
\hline G3B & 2458.33 & 15.02 & 940.00 & 2820.00 & 7059922.24 & 18453.16 \\
\hline G4A & 4860.96 & 26.24 & 940.00 & 2820.00 & 7424593.88 & 19862.04 \\
\hline G4B & 4917.64 & 25.00 & 940.00 & 2820.00 & 7384079.42 & 21204.14 \\
\hline G5A & 4400.93 & 17.42 & 940.00 & 2820.00 & 7505326.89 & 26793.34 \\
\hline G5B & 3115.96 & 10.3 & 940.00 & 2820.00 & 7384079.42 & 32610.63 \\
\hline G6A & 2850.26 & 10.12 & 940.00 & 2820.00 & 7220927.9 & 31046.43 \\
\hline G6B & 3143.82 & 10.48 & 940.00 & 2820.00 & 7438076.25 & 32102.24 \\
\hline
\end{tabular}




\subsubsection{CÁLCULO DE LA DUCTILIDAD AL DESPLAZAMIENTO $\mu \mu$.}

Este parámetro mide la relación entre el desplazamiento máximo y el desplazamiento en el límite proporcional a partir del cual se genera el punto de primera cadencia del material y cambia la pendiente de la recta tangente de la gráfica esfuerzo vs. Deformación dando inicio al rango plástico del material en el cual ya existen deformaciones permanentes. (Tabla 9)

El cálculo de la ductilidad al desplazamiento se realiza con la siguiente ecuación.

$\mu \Delta=$ Ductilidad al desplazamiento.

$$
\mu \Delta=\frac{\Delta u}{\Delta y}
$$

$\Delta \mathrm{u}=$ Desplazamiento máximo.

$\Delta \mathrm{y}=$ Desplazamiento máximo en el límite proporcional.

Nótese que en un comportamiento frágil el valor de ductilidad al desplazamiento tiende a 1.

Tabla 9.

Indice de ductilidad.

\begin{tabular}{|c|c|c|c|}
\hline Probeta & $\Delta$ máximo $(\mathbf{m m})$ & $\begin{array}{c}\Delta \text { límite proporcional } \\
(\mathbf{m m})\end{array}$ & $\mu \Delta$ \\
\hline G1A & 100 & 37.72 & 2.65 \\
\hline G1B & 46.84 & 39.19 & 1.20 \\
\hline G2A & 33.28 & 14.7 & 2.26 \\
\hline G2B & 19.49 & 6.74 & 2.89 \\
\hline G3A & 47.64 & 7.26 & 6.56 \\
\hline G3B & 31.69 & 15.02 & 2.11 \\
\hline G4A & 67.69 & 26.24 & 2.58 \\
\hline G4B & 50.35 & 25.00 & 2.01 \\
\hline G5A & 36.66 & 17.42 & 2.10 \\
\hline G5B & 51.34 & 10.30 & 4.98 \\
\hline G6A & 13.99 & 10.12 & 1.38 \\
\hline G6B & 40.304 & 10.48 & 3.85 \\
\hline
\end{tabular}

\section{ANÁLISIS DE RESULTADOS}

Los valores de cortante, momento flector y deflexión para la carga en el límite proporcional y la carga máxima presentan una alta dispersión en los resultados, esto se ve reflejado en la gran reducción de la resistencia en el valor característico del Módulo de ruptura y el esfuerzo de la fibra en el límite proporcional del sistema propuesto.

Por otro lado, la dispersión en los valores de esfuerzo en la fibra en el límite proporcional fue la mayor de todos los datos recolectados, este comportamiento se debe a que fue difícil garantizar la calidad en toda la línea de encolado por las irregularidades que presenta la esterilla. En el momento en que el patín superior, sometido a esfuerzos de compresión, empieza deformarse y a despegarse de la madera termina el rango elástico del material, entonces la carga máxima al límite proporcional está directamente relacionada con la calidad del adhesivo sobretodo en el patín superior.

Los valores del módulo de elasticidad tuvieron un coeficiente de variación del $20 \%$, teniendo la menor dispersión de todos los resultados. A continuación, se presenta el resumen de los valores promedio, el valor para el quinto percentil y el valor característico. (Tabla 10) 
Tabla 10.

Indice de ductilidad.

\begin{tabular}{|c|c|c|c|}
\hline & PROMEDIO & $\begin{array}{c}\text { QUINTO } \\
\text { PERCENTIL }\end{array}$ & $\begin{array}{c}\text { VALOR } \\
\text { CARACTERÍSTICO }\end{array}$ \\
\hline Sft(MPa) & 30.26 & 14.77 & 7.72 \\
\hline SFc(MPa) & 22.74 & 10.49 & 4.94 \\
\hline SRt $(\mathrm{MPa})$ & 39.00 & 17.94 & 11.36 \\
\hline SRc $(\mathrm{MPa})$ & 29.1 & 12.77 & 7.56 \\
\hline $\mathrm{E}(\mathrm{MPa})$ & 28299.23 & 19228.04 & 16219.06 \\
\hline
\end{tabular}

\subsection{RELACIÓN ENTRE EL CH DE LAS PIEZAS DE MADERA REUTILIZADA Y EL COMPORTAMIENTO MECÁNICO DE LAS VIGAS.}

Se realizan gráficos relacionando la resistencia y el módulo de elasticidad con el contenido de humedad $\mathrm{CH}$ de la madera reutilizada en donde se presenta la falla y la desviación estándar de los contenidos de humedad de las piezas, como parámetro para determinar la influencia de la variación entre los contenidos de humedad de la madera que compone la viga. (figura 9)
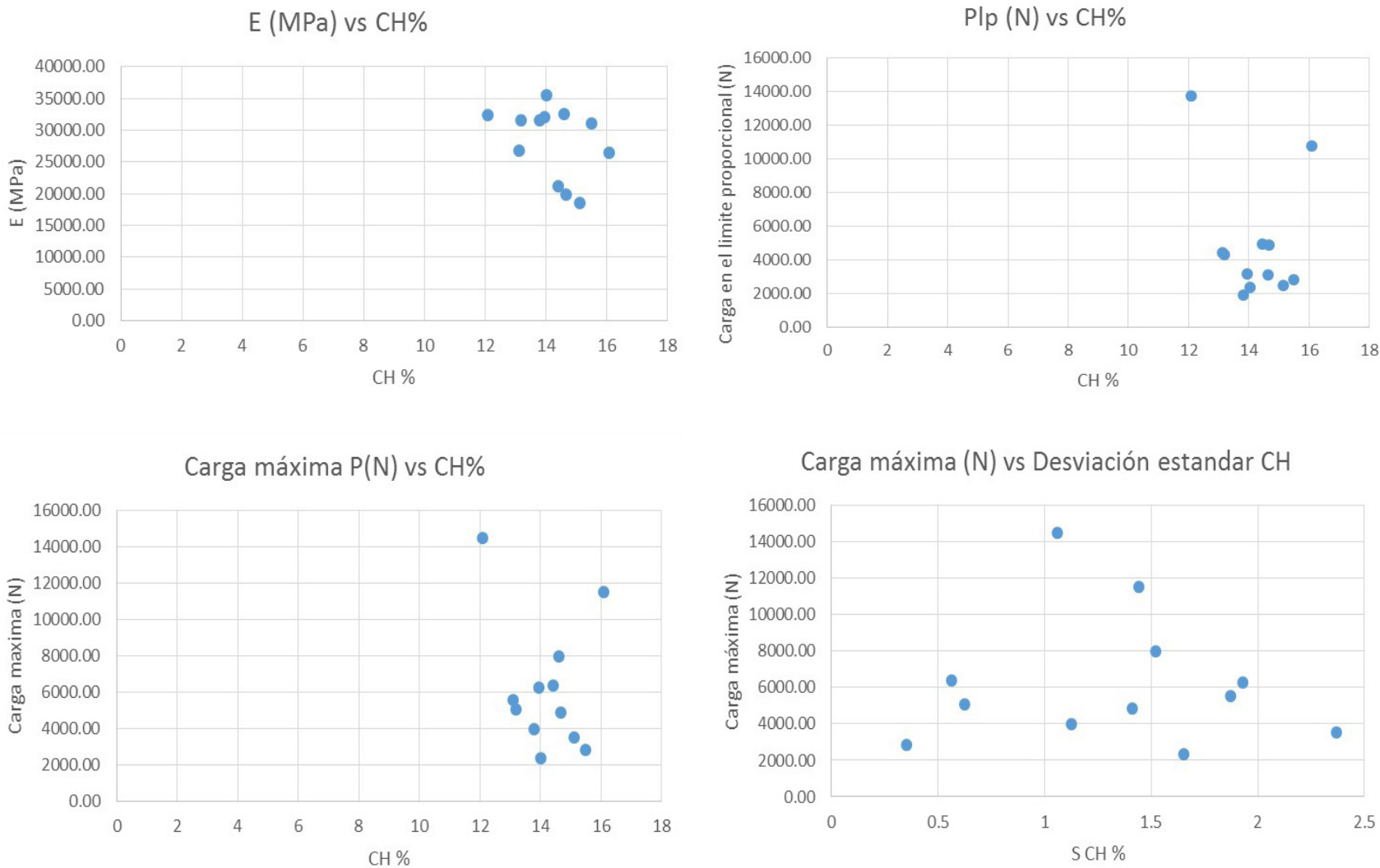

Figura 9. Módulo de elasticidad, carga en el limite proporcional, carga maxima vs $\mathrm{CH}$ de la pieza de madera en el lugar de la falla.

No se encuentra una tendencia clara entre la relación del contenido de humedad de la madera reutilizada y el módulo de elasticidad de las vigas, lo mismo ocurre entre el contenido de humedad y la carga máxima registrada en el ensayo de flexión. Al analizar la variación de los contenidos de humedad (desviación estándar del $\mathrm{CH}$ ) de las piezas que componen las vigas con relación a la carga máxima tampoco se encuentra una tendencia definida. El comportamiento de las vigas no está determinado por el contenido de humedad de las piezas que componen el alma de las probetas ensayadas. Siempre y cuando se garantice la calidad de la línea de cola. 


\subsection{RELACIÓN ENTRE EL TAMAÑO DE LAS PIEZAS DE MADERA REUTILIZADA Y EL COMPORTAMIENTO MECÁNICO DE LAS VIGAS}

A pesar de que las vigas con piezas de $0.65 \times 0.16 \mathrm{~m}$. tienen una carga máxima mayor que las de 0.87 $x 0.16 \mathrm{~cm}$. Se puede ver una tendencia en el aumento de la resistencia con relación al tamaño máximo de las piezas que componen la viga. Las vigas con piezas de $1.30 \times 0.16 \mathrm{~m}$. fueron las que soportaron la mayor carga $\mathrm{y}$ las piezas de $0.87 \times 0.16 \mathrm{~cm}$ la menor. (figura 10)

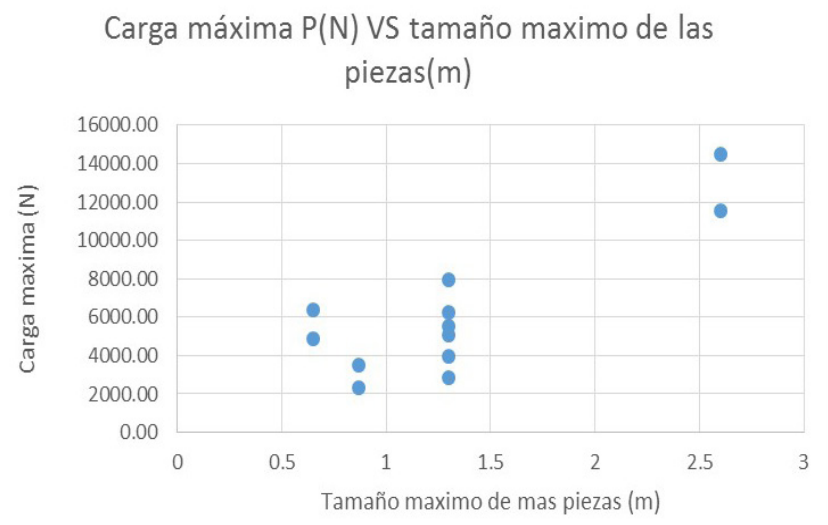

Figura 10. Carga máxima vs tamaño de las piezas de madera reutilizada.

Durante los ensayos de flexión se observó que existe un desplazamiento entre las piezas de madera que componen el alma de la viga, es por esto que se analiza la relación entre el módulo de elasticidad y la cantidad de juntas que se presentan en cada viga, además de la relación entre el comportamiento de la carga máxima resistida respecto al número de uniones. (figura 11)
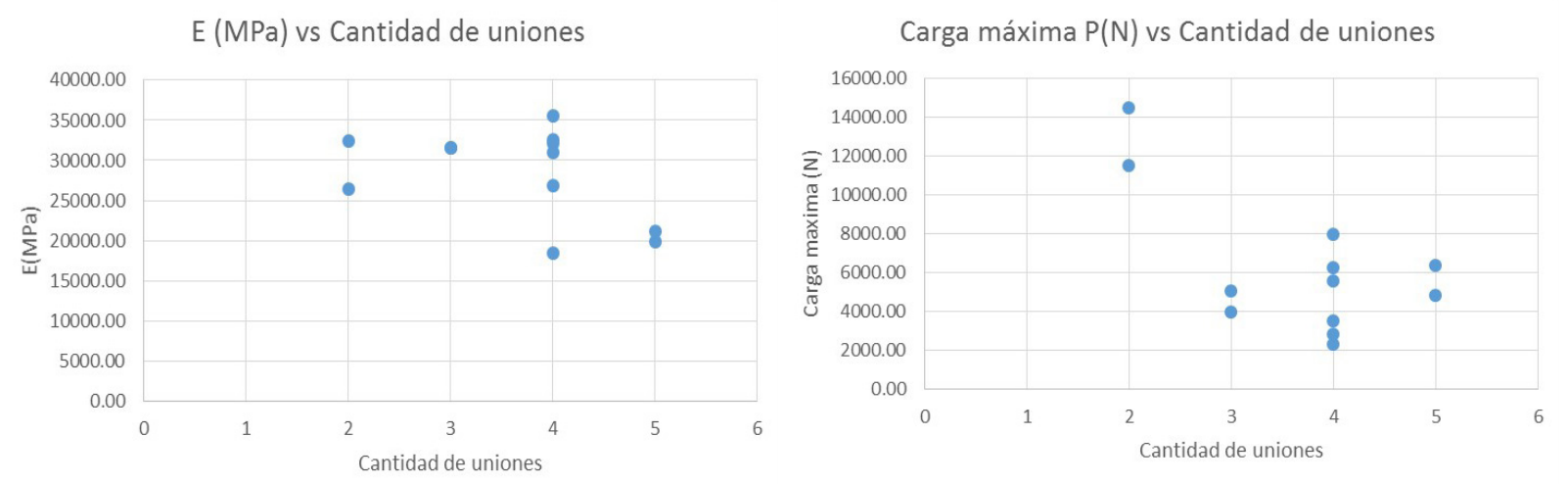

Figura 11. Módulo de elasticidad y Carga maxima vs Cantidad de uniones

En vigas con tres uniones se presenta la menor dispersión de resultados, la carga máxima se registró en vigas con dos uniones, sin embargo, el módulo de elasticidad máximo se presentó en vigas de cuatro uniones. El mejor comportamiento se obtiene en vigas con dos uniones, siendo estas vigas las que tienen la menor cantidad de juntas de todas las vigas analizadas.

\subsection{RELACIÓN ENTRE LA DENSIDAD DE PIEZAS DE MADERA REUTILIZADA Y EL COMPORTAMIENTO MECÁNICO DE LAS VIGAS}

Se halló el porcentaje de madera de alta densidad de cada probeta y se realizaron gráficos de porcentaje de piezas de alta densidad versus la carga máxima y el módulo de elasticidad. La carga máxima se logró en para $87 \%$ de maderas de alta densidad en el alma, sin embargo, hay gran variabilidad entre los valores de carga máxima para este porcentaje, no se encuentra una tendencia entre la resistencia de las vigas y la densidad de las maderas que componen el alma de la viga. (figura 12) 
Carga máxima $\mathrm{P}(\mathrm{N})$ vs \% madera de alta densidad

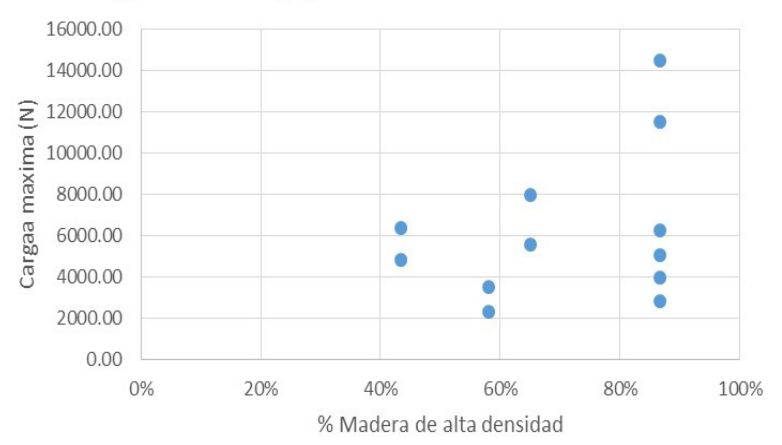

Figura 12. Carga máxima vs porcentaje de madera de alta densidad.

En cuanto al módulo de elasticidad, el valor máximo y el mínimo se reportó para $65 \%$ de maderas de alta densidad, la menor dispersión en los resultados se logró con $87 \%$ de maderas de alta densidad. No se establece una tendencia clara entre el módulo de elasticidad de la viga y la densidad de las piezas de madera reutilizada que compone el alma de la viga. (figura 13)

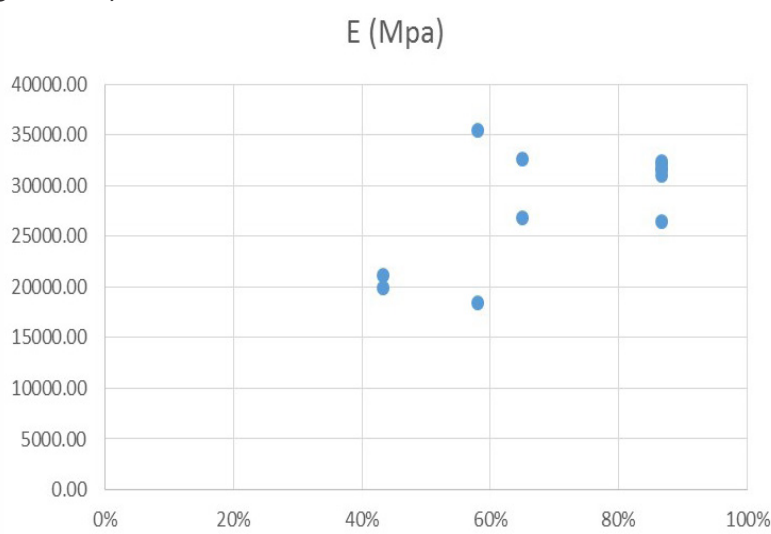

Figura 13. Módulo de elasticidad vs porcentaje de madera de alta densidad.

\subsection{TIPOS DE FALLA}

\subsubsection{TIPO DE FALLA 1: COMPORTAMIENTO FRÁGIL. FALLA EN LA LÍNEA DE COLA}

En este tipo de falla la viga llega a un nivel de esfuerzos en el cual la esterilla de guadua del patín inferior se separa del adhesivo (falla en la línea de cola), este tipo de falla se debe a defectos de en la fabricación en el proceso de encolado y prensado, también ocurre cuando no se retira completamente el tejido parenquimático de la guadua. (figura 14)

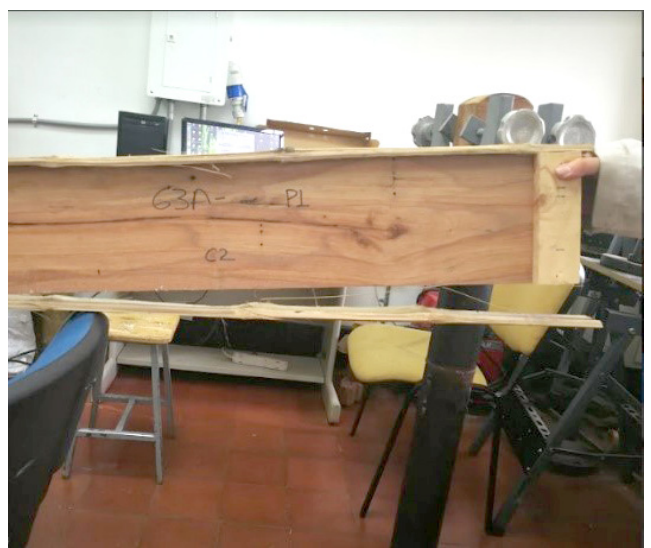

Figura 14. Separación de la guadua del patin inferior en un extremo de la viga. 


\subsubsection{COMPORTAMIENTO DÚCTIL: FALLA POR TENSIÓN EN LAS FIBRAS DEL PATÍN INFERIOR}

Este tipo de falla fue común en la mayoría de las probetas ensayadas, al empezar a aumentar la carga las vigas tienen un comportamiento elástico y empiezan a deformarse las fibras del patín superior que no están adheridas a la madera reutilizada. (figura 15)

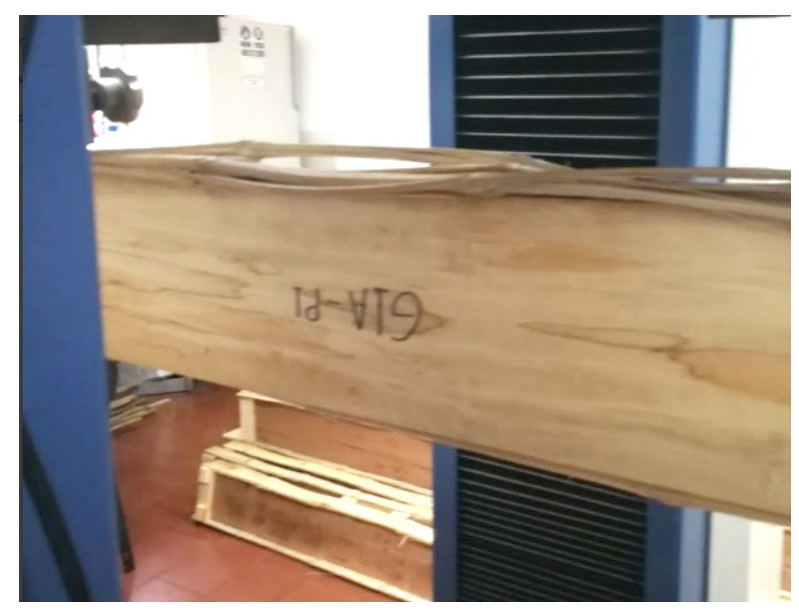

Figura 15. Deformacion fibras del patin superior.

En esta etapa hay un desplazamiento relativo entre las piezas de madera reutilizada, esta deformación solamente es controlada por la esterilla de guadua. La guadua realiza el mayor aporte a la rigidez de la viga. (figura 16)

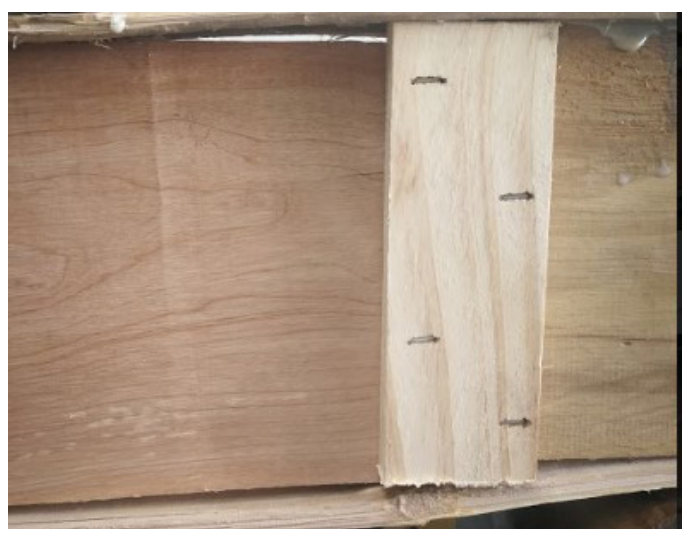

Figura 16. Desplazamiento relativo piezas de madera reutilizada.

Al aumentar la carga y la deflexión, la esterilla de la parte superior empieza a despegarse de las piezas de madera, en este punto se registra el final del comportamiento elástico de la viga, el inicio del comportamiento plástico se caracteriza por que las fibras de guadua del patín inferior empiezan a ser más solicitadas. Ya hay deformaciones permanentes. (figura 17)

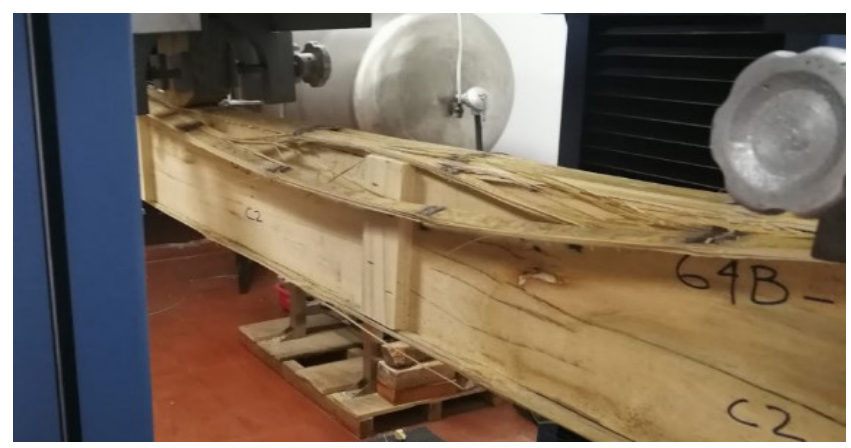

Figura 17. Separación de la guadua del patin superior. 
Las deformaciones continúan y existen saltos en la gráfica carga deformación a medida que se despegan las fibras del patín superior, sin embargo, en todos los casos la viga continúa asumiendo carga hasta que supera la carga obtenida en el límite proporcional. (figura 18)

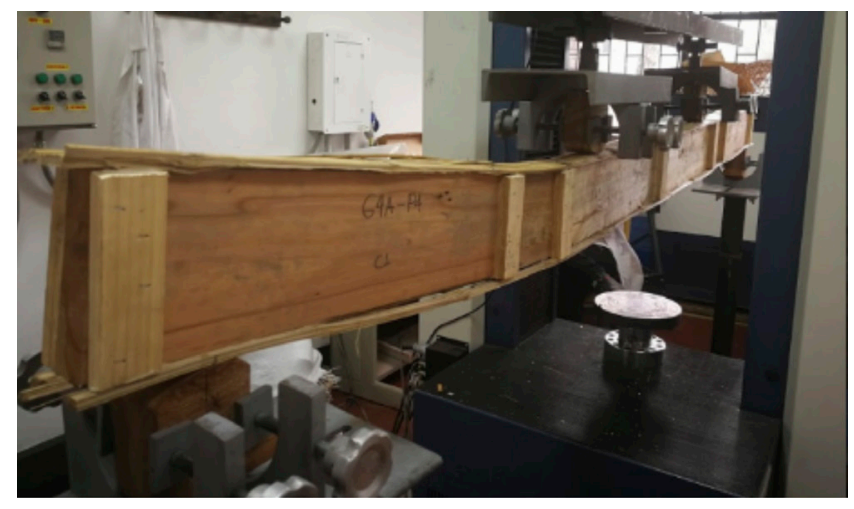

Figura 18. Esfuerzos asumidos por el patin inferior.

Las deformaciones empiezan a ser mayores para pequeños incrementos de carga sin fallar (ductilidad), la falla se produce cuando se pierde la adherencia entre el patín inferior y el adhesivo o cuando las fibras de guadua superan su resistencia a la tensión. Las piezas de madera reutilizada no sufrieron daños en ninguna viga y se pueden reutilizar nuevamente. (figura 19)

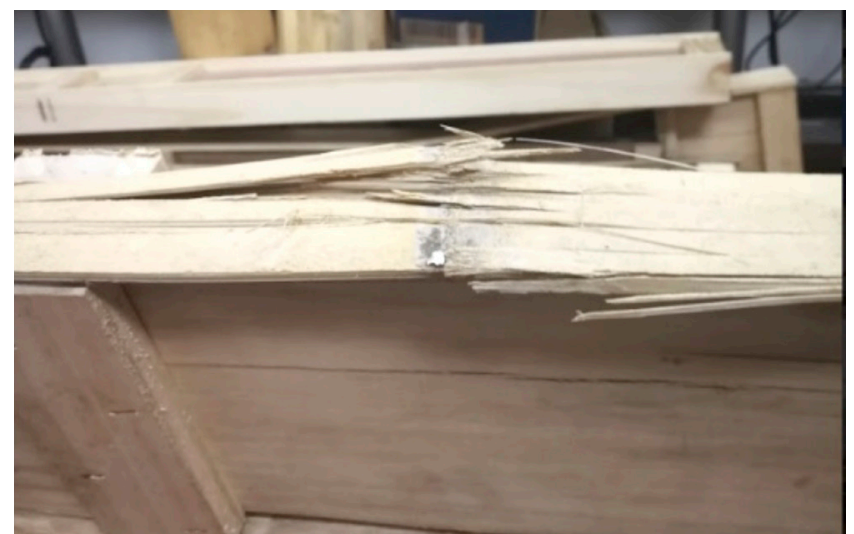

Figura 19. Falla por tension de las fibras del patin inferior.

\subsection{ANÁLISIS DE LA DUCTILIDAD}

En general las vigas tienen un comportamiento dúctil, al finalizar su comportamiento elástico, las vigas continúan deformándose plásticamente sin colapsar. En todas las vigas se produce la carga máxima en el rango plástico. (figura 20)

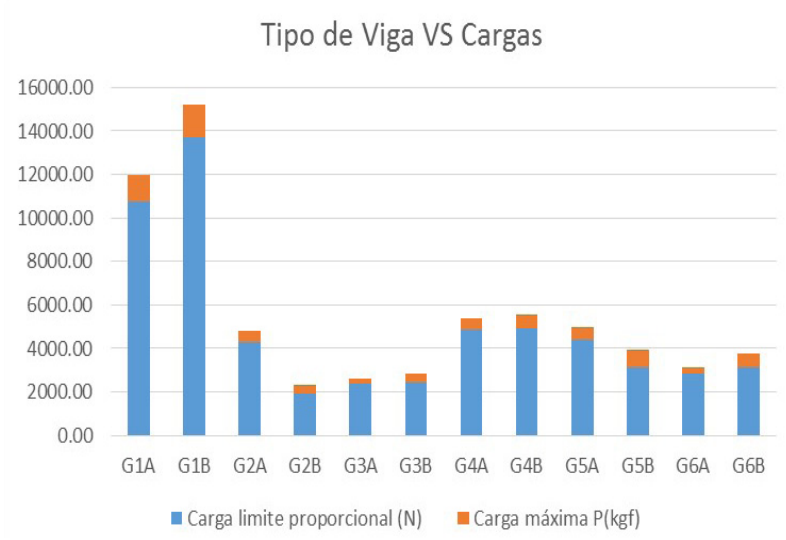

Figura 20. Tipo de viga vs carga maxima y carga en el limite proporcional. 
Este comportamiento es producido por la ductilidad de la Guadua angustifolia Kunth, esta ductilidad se debe a que a ciertos niveles de esfuerzo empiezan a romperse gradualmente algunas fibras, sin embargo, existe una redistribución de esfuerzos en las fibras que no han fallado. (Estrada, 2016)

La ductilidad máxima al desplazamiento ocurrió en la viga G3A, para esta viga se obtuvo un valor de 6.56, La viga puede deformarse 6.5 veces más que la deformación alcanzada en el límite proporcional, el menor valor registrado fue de 1.20 en la viga G1B, esta viga presento el mayor valor de carga máxima.

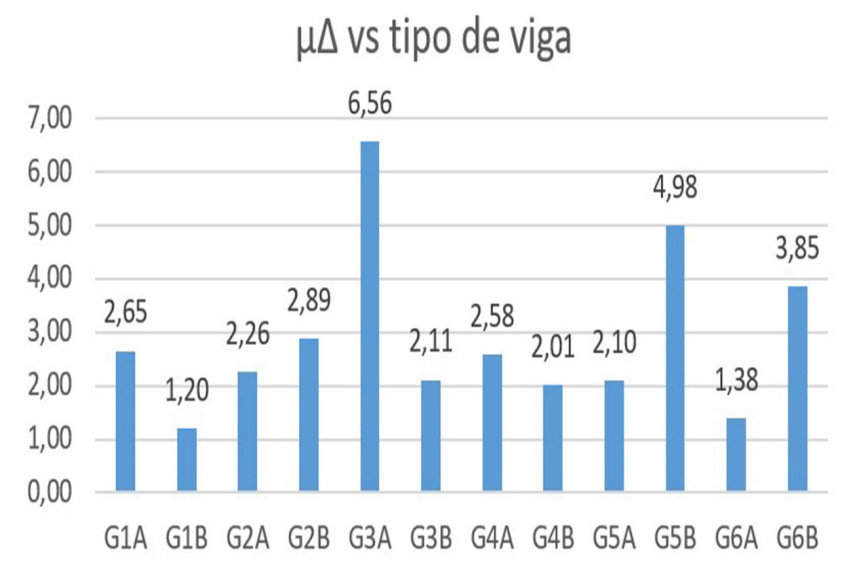

Figura 21. Ductilidad al desplazamiento vs tipo de viga.

El valor promedio del parámetro de ductilidad al desplazamiento es de 2.88, con una desviación estándar de 1.55, el coeficiente de variación es $54 \%$. (figura 21 )

\section{CONCLUSIONES Y RECOMENDACIONES}

Teniendo en cuenta el impacto ambiental que produce la industria de la construcción, es necesario desarrollar prácticas sostenibles, la innovación en materiales de construcción se desarrolla en un contexto de reducción de extracción de materias primas no renovables y mitigación de impactos ambientales ocasionados por deshechos de construcción. En países que no han desarrollado tecnologías basadas en la reutilización, los materiales se deben someter a procesos de transformación para ser reutilizados. Esta investigación propuso la construcción de elementos estructurales a partir de madera reutilizada unida mediante Guadua angustifolia Kunth que produjo las siguientes conclusiones:

\section{ETAPA DE FABRICACIÓN:}

- Procesamiento de la madera reutilizada:

Las piezas de madera reutilizada se deben someter a una exhaustiva inspección visual, es importante analizar patológicamente cada pieza de madera recuperada para identificar lesiones físicas, mecánicas o químicas que puedan afectar el comportamiento mecánico de las vigas. Durante el proceso de desmonte, las piezas de madera sufren perforaciones, agrietamiento, aristas faltantes y contaminación por agentes químicos. Estos defectos deben ser retirados manteniendo el mayor tamaño posible de cada pieza. Se deben ajustar las dimensiones de cada elemento garantizando uniformidad en la altura, el ancho y el espesor de las piezas de madera, garantizando el contacto total entre elementos adyacentes, teniendo en cuenta que diferentes medidas en la altura de las piezas ocasiona que la presión de prensado no sea uniforme a lo largo de la viga reduciendo la resistencia de la línea de cola. 
- Procesamiento de la Guadua angustifolia Kunth:

Las fibras de esterilla de guadua deben ser continuas a lo largo de toda la viga, es difícil controlar el ancho de la esterilla, por este motivo se debe garantizar que la menor medida del ancho corresponda a la medida de diseño. El tejido parenquimático debe ser retirado hasta que las fibras de la guadua queden expuestas, las protuberancias de los nudos deben ser pulidas hasta que toda la esterilla tenga el mismo espesor, de no ser así la presión de prensado se concentrará en estos puntos generando deficiencias en la presión de prensado en los entrenudos. En los casos en que en la superficie exterior de la esterilla se encuentren ramas, se deben retirar dejando la superficie lo más uniforme posible, de no ser retiradas se presentan problemas en el prensado ya que se genera una rotación del elemento y es imposible realizar este procedimiento.

- Proceso de encolado, prensado y curado:

Se deben seguir las recomendaciones del fabricante en cuanto la dosificación de adhesivo y catalizador adecuada, además de la presión de prensado y el tiempo de curado correcto. Antes de iniciar en encolado se debe verificar que las superficies de las piezas de madera y de guadua estén limpias y sean uniformes, el adhesivo se debe aplicar de manera uniforme evitando excesos.

- Resultados obtenidos:

La mayor carga máxima registrada en los ensayos de flexión fue de $14482.00 \mathrm{~N}$, para la viga G1B compuesta por dos piezas de madera de baja densidad en los extremos $(0.2 \mathrm{~m} \times 0.16 \mathrm{~m})$ y un elemento central de madera de alta densidad $(2.60 \mathrm{~m} \times 0.16)$, esta configuración presenta el menor número de uniones y la pieza de madera de mayor longitud.

La menor carga máxima registrada fue de $2353.30 \mathrm{~N}$, para la viga G3A compuesta por dos elementos de alta densidad en los extremos $(0.87 \mathrm{~m} \times 0.16 \mathrm{~m})$, y tres elementos centrales de baja densidad: un elemento central de $(0.87 \mathrm{~m} \times 0.16 \mathrm{~m})$ y dos elementos a cada lado de $(0.2 \mathrm{~m} \times 0.16 \mathrm{~m})$.

El esfuerzo de la fibra más alejada del eje neutro en el límite proporcional y el módulo de rotura se calcularon para las fibras sometidas a tensión y a compresión, los máximos esfuerzos se producen en las fibras inferiores sometidas a tensión. Al tratarse de un elemento compuesto por varios materiales fue necesario calcular la relación entre el módulo de elasticidad de la guadua y una constante de elasticidad en el alma. Teniendo en cuenta que las maderas reutilizadas solo están unidas en la parte superior e inferior por guadua, la fricción que se desarrolla entre las piezas adyacentes no es suficiente para asumir el esfuerzo de corte y se presenta un desplazamiento relativo entre piezas de madera.

Para encontrar la relación entre el módulo de elasticidad de la guadua y el alma de la viga y poder calcular la geometría de la sección transformada se analizó la deformación de la viga mediante el método de los elementos finitos, realizando iteraciones hasta conseguir la deflexión registrada en el ensayo de flexión en una condición de carga determinada dentro del rango elástico.

La dispersión de los resultados fue bastante alta, para el esfuerzo de la fibra más alejada del eje neutro en el límite proporcional se tiene un coeficiente de variación de $61.24 \%$ en tensión y $67.85 \%$ a compresión. En cuanto al módulo de ruptura el coeficiente de variación es de $47.01 \%$ a tensión y $52.37 \%$ a compresión. Los valores del módulo de elasticidad tuvieron un coeficiente de variación del $20 \%$, teniendo la menor dispersión de todos los resultados.

La dispersión alta hace que los valores promedio de esfuerzo de la fibra más alejada del eje neutro en el límite proporcional y módulo de ruptura se reduzcan en más de un 50\% para el quinto percentil y en más del $70 \%$ para el valor característico. En el caso del módulo de elasticidad se reduce en un $32 \%$ para el quinto percentil y $43 \%$ para 
el valor característico. La dispersión en los resultados se produce por la variación en la calidad en el proceso de encolado teniendo en cuenta que las irregularidades en el espesor de la esterilla provocan deficiencias de presión en algunas zonas, así mismo es difícil mantenerla en su sitio justo antes de aplicarles presión.

La variación de los contenidos de humedad (desviación estándar del $\mathrm{CH}$ ) de las piezas que componen las vigas con relación a la carga máxima no muestra una tendencia definida. El comportamiento de las vigas no está determinado por el contenido de humedad de las piezas que componen el alma de las probetas ensayadas.

No se encontró una relación entre el contenido de humedad de las piezas de madera reutilizada en donde ocurre la falla y la resistencia de las vigas, lo mismo ocurre con el tamaño máximo de las piezas utilizadas y con el número de uniones. El comportamiento depende entonces de la resistencia los elementos de Guadua angustifolia Kunth y la calidad de la línea de adhesivo.

En vigas con tres uniones se presenta la menor dispersión de resultados, la carga máxima se registró en vigas con dos uniones, sin embargo, el módulo de elasticidad máximo se presentó en vigas de cuatro uniones. El mejor comportamiento se obtiene en vigas con dos uniones, siendo estas vigas las que tienen la menor cantidad de juntas de todas las vigas analizadas.

En cuanto a la relación entre el módulo de elasticidad y la densidad de la madera reutilizada, el valor máximo y mínimo del módulo de elasticidad se reportó para las vigas construidas con el $65 \%$ de maderas de alta densidad, la menor dispersión en los resultados se logró con $87 \%$ de maderas de alta densidad. No se establece una tendencia clara entre el módulo de elasticidad de la viga y la densidad de las piezas de madera reutilizada que compone el alma de la viga.

Las vigas tienen un comportamiento dúctil, al finalizar su comportamiento elástico, las vigas continúan deformándose plásticamente $\sin$ fallar. En todas las vigas se produce la carga máxima en el rango plástico. La ductilidad máxima al desplazamiento ocurrió en la viga G3A, para esta viga se obtuvo un valor de 6.56 , lo cual quiere decir que puede deformarse 6.5 veces más que la deformación alcanzada en el límite proporcional, el menor valor registrado fue de 1.20 en la viga G1B, esta viga presento el mayor valor de carga máxima.

- Tipos de falla:

Se registran dos tipos de falla principales:

Falla frágil cuando se falla la línea de adhesivo del patín inferior en los extremos de las vigas debido a defectos en la fabricación específicamente en el proceso de encolado y prensado.

Falla dúctil en la cual se presenta un comportamiento elástico en el cual las fibras superiores sometidas a compresión empiezan a deformarse en las zonas que no están adheridas al alma y se producen desplazamientos relativos entre las piezas, luego el patín superior empieza a despegarse del alma empezando el comportamiento elástico de la viga, este proceso es gradual y produce saltos en la curva carga vs deformación, la deformación empieza a aumentar para pequeñas variaciones de carga porque todo el esfuerzo lo asume el patín inferior, la falla se produce cuando la guadua de la parte inferior falla por tensión o cuando se despega del alma en los extremos. Ninguna pieza de madera reutilizada sufrió daños, la ruptura se registra en la esterilla de guadua o en la línea de cola.

\section{- RECOMENDACIONES}

Es importante buscar una menor dispersión en el comportamiento mecánico de las vigas de madera reutilizada unidas con Guadua angustifolia Kunth para aumentar los valores característicos. Teniendo en cuenta que hay vigas que tuvieron un buen comportamiento hay que mejorar el proceso de fabricación de las piezas de esterilla de 
Guadua angustifolia Kunth aumentando la calidad de la línea de adhesivo, garantizando una presión de prensado uniforme en toda la viga.

La selección de la esterilla se realizó bajo la premisa de trabajar con el material que generara el menor consumo energético y el menor costo económico. Sin embargo, para evitar problemas en el proceso de prensado, se deben incorporar procesos y equipos que permitan trabajar con productos de guadua más uniformes. Las latillas de guadua solucionan problemas en el prensado ya que las superficies son uniformes, sin embargo, se pierde el aporte de la pared externa que es la más resistente debido a su dureza y mayor cantidad de fibras.

A pesar de ser un elemento construido con madera presenta ductilidad gracias a la guadua, se recomienda utilizar un método de diseño estructural más adecuado ya que en el método de los esfuerzos admisibles, por el cual se diseñan las estructuras de madera según el reglamento colombiano de construcción sismo resistente NSR10, no se tendría en cuenta la ductilidad las vigas estudiadas.

\section{DISCUSIÓN}

Es necesario incentivar la práctica del reciclaje y la reutilización de materiales que han sido desechados en actividades de constricción, en esta investigación se proponen elementos estructurales innovadores, teniendo en cuenta que reutilizan madera uniéndola con Guadua angustifolia Kunth, un material con grandes ventajas ambientales y mecánicas.

Sin embargo, para reducir al máximo el consumo de energía en el proceso de fabricación se decidió utilizar esterilla de guadua, lo cual ocasionó inconvenientes en la fase de encolado y prensado de las vigas, que se vio reflejado en una alta dispersión de los resultados, esta dispersión conlleva a que los valores característicos de los parámetros que determinan el comportamiento mecánico de las vigas sean mucho menores a los valores promedio encontrados.

Para disminuir la dispersión de los resultados se propone mejorar el proceso de fabricación de los elementos de Guadua angustifolia Kunth, aunque se consumirá más energía en el proceso se podrían obtener valores característicos más altos que permitan optimizar el uso de estos elementos en estructuras.

Hay que destacar que el sistema propuesto tiene un comportamiento dúctil con un rango inelástico bastante amplio, por lo tanto, es un elemento estructural que acumula una gran cantidad de energía inelástica de deformación, un estudio detallado del módulo de tenacidad determinará la energía necesaria para llegar al punto de ruptura, este parámetro es sumamente importante para el diseño de construcciones sismo resistentes. Sin embargo, para aprovechar la ductilidad del material el diseño estructural no debe realizarse por el método de los esfuerzos admisibles.

El sistema propuesto aporta significativamente a la cadena de valor de la Guadua angustifolia Kunth, que a pesar de ser un recurso abundante ha sido subvalorado, el desarrollo científico y la innovación en productos a base de este material que promuevan alternativas sostenibles dinamizarán la economía rural contribuyendo al desarrollo social de las comunidades. 


\section{REFERENCIAS}

Environmental Protection Agency United States, (2008). "Lifecycle Construction Resource Guide".

McDonoufh, W. \& Braungart, M. (2002). " Cradle to cradle: Remaking the way we make things.

Guy, A. (2002). "Design for Deconstruction and Materials Reuse.”

Zabusova, D. (2014). "Design for Deconstruction"

Flak, R., Green, D. \& Lantz, S. (1999). "Evaluation of lumber recycled from an industrial military building" vol. 49, no. 8816 , pp. $49-55$.

Zhong, R., Gu, J., Gao, Z., Tu, D. \& Hu, C. (2017). "International Journal of Adhesion and Adhesives Impacts of urea-formaldehyde resin residue on recycling and reconstitution of wood-based panels," vol. 78, no. May, pp. $60-66$.

Pérez, V. (1992). "Manual de madera laminada" Instituto forestal de Chile. Santiago.

Quevedo, V. (2000). "Adherencia y adhesivos para madera"

Olarte, A. (2012). "Diseño de conexiones en elementos estructuales de bambú - Guadua laminada pegada prensada para un proyecto de vivienda".

Carvajal, W., Ortegon, W. \& Romero, C. (1981). "Elementos estructurales en bambú".

Lozano, J. (2010). “Diversas aplicaciones del bambú en la arquitectura”.

Gonzalez, H., Hellwing, S. \& Montoya, J. (2009) "Determinación del adhesivo óptimo en la fabricación de vigas de bambú (Guadua angustifolia) procedure to determine the optimum adhesive for manufacturing timber of bamboo (Guadua angustifolia)".

Estrada, M. (2016). "Modelo numérico micromecánico del proceso de fractura de estructuras fabricadas con bambú Guadua angustifolia".

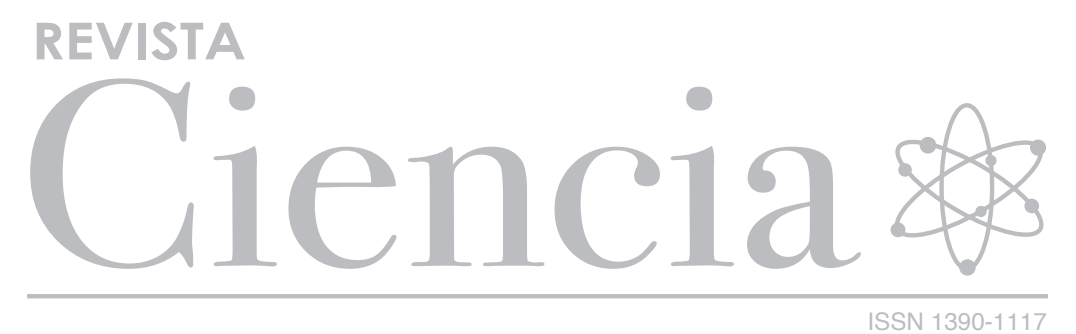

(C) Universidad de las Fuerzas Armadas ESPE Sangolquí, Ecuador.

CIENCIA - QUITO, Volumen 22, No. 1, Enero 2020 